\title{
Vigilância Alimentar e Nutricional: Antecedentes, Objetivos e Modalidades. A VAN no Brasil
}

\section{Food and Nutritional Surveillance in Brazil: Background, Objectives and Approaches}

\author{
Malaquias Batista-Filho ${ }^{1} \&$ Anete Rissin ${ }^{2}$
}

\begin{abstract}
BATISTA-FILHO, M. \& RISSIN, A. Food and Nutritional Surveillance in Brazil: Background, Objectives and Approaches. Cad. Saúde Públ., Rio de Janeiro, 9 (supplement 1): 99-105, 1993.

The authors describe the concepts, objectives, components and strategic actions that are most frequently recommended for the development of systems of food and nutritional surveillance. The study outlines the importance of a continuous process of data collection, processing and analysis for the follow-up of food and nutritional trends. The System for Food and Nutritional Surveillance could be a valuable instrument for the planning, implementation and analysis of programs and policies of many international agencies of health and nutrition for the year 2000 (UNICEF/FAO/WHO). The study analyzes the evolution and current situation of the food and nutritional surveillance systems in Brazil, and a realtionship is drawn between its main problems (coverage, indicators, usage and analytical capacity) for the consolidation of the process.
\end{abstract}

Key words: Nutritional Surveillance; Food

\section{ANTECEDENTES HISTÓRICOS}

O lançamento, há 50 anos, do livro de Josué de Castro - Geopolítica da Fome - e a realização, nas décadas de 50 e 60, de vários inquéritos sobre a situação alimentar e nutricional de muitos paises da África, Ásia e América Latina despertaram a consciência mundial para o grave quadro das deficiências globais e específicas de alimentos/nutrientes. Até então, estes problemas tinham sido abordados de uma forma impressionista, sem um tratamento técnico-científico que possibilitasse compreender sua natureza, extensão, gravidade e distribuição populacional e geográfica (OPS, 1989).

Manifesta-se, desde então, um esforço multinacional no sentido de definir políticas e programas sociais orientados para a redução das

\footnotetext{
${ }^{1}$ Instituto Materno-Infantil de Pernambuco. Rua dos Coelhos, 300, Boa Vista. Recife, PE, 50070-550, Brasil ${ }^{2}$ Fundação Nacional de Saúde-PE. Avenida Conselheiro Rosa e Silva, 1489, Tamarineira. Recife, PE, 52050-020, Brasil.
}

deficiências alimentares, dos agravos nutricionais e suas conseqüências no quadro biológico e social. Evidencia-se, mediante repetidas experiências, que os dados produzidos pelos clássicos inquéritos nutricionais (ICNND, 1963; Jelliffe, 1968) eram de pouca utilidade para o monitoramento das propostas de intervenção, na medida em que exigia-se a participação de profissionais de elevada qualificação, equipamentos sofisticados e altos custos financeiros (Chavez, 1989; Lechtig, 1990). Com estas restrições, os estudos de base populacional dificilmente poderiam ser repetidos a intervalos menores que 10 ou 15 anos.

$\mathrm{O}$ interesse político pelo encaminhamento de medidas destinadas a reduzir o gap existente entre o potencial de recursos naturais e humanos e o perfil de qualidade de vida de regiões, países e agrupamentos sociais com escassa participação no sistema de produção e uso de bens e serviços consolidou-se, a partir dos anos 70, com a realização de três eventos internacionais: a Conferência Mundial de Alimentos, promovida pela Food and Agriculture Organization (FAO), em Roma (1974); a Conferência de 
Alma-Ata, na União Soviética, em 1978, auspiciada pela Organização Mundial da Saúde; e a proposta de uma Revolução pela Sobrevivência e Desenvolvimento da Criança, patrocinada pela United Nations Children's Fund (Unicef) (1983).

Os compromissos formais assumidos nestas três assembléias são complementares entre si. Em função da primeira, estabelece-se a proposta de assegurar o acesso aos alimentos a toda a população humana, com ênfase nos grupos biológicos de elevado risco (FAO, 1974); a segunda propõe metas e estratégias para o atendimento universal às necessidades básicas de saúde, privilegiando, mais uma vez, mães e crianças (OMS, 1978); por fim, a proposta do Unicef representa um avanço qualitativo em relação à saúde da criança, objetivando, mais do que a simples redução da mortalidade, o alcance de condições adequadas de desenvolvimento biológico e social. (Unicef, 1990).

As implicações práticas condicionadas por tais compromissos confluem, no caso da alimentação e da nutrição, para um denominador comum: a necessidade de se assegurar mecanismos ágeis de informação e decisão que possibilitem, de forma competente, o acompanhamento da situação e suas tendências, bem como a avaliação da pertinência e eficácia das ações empreendidas (Mason, 1984).

É sob este enfoque que se define o papel dos sistemas de vigilância alimentar e nutricional, concebidos como um processo de geração e análise sistemática de informações sobre a situação alimentar e nutricional e seus fatores determinantes. Tais sistemas justificam-se quando, de forma consequiente, oferecem subsídios para a formulação de políticas, desenho de programas e resgate crítico de seus resultados, gerando o feedback necessário para o aperfeiçoamento contínuo das medidas de intervenção (Aranda-Pastor \& Kevany, 1980).

\section{OBJETIVOS E COMPONENTES}

A vigilância alimentar e nutricional (VAN) é um conceito unicista, na medida em que se propõe a reunir elementos para a definição de políticas e para a instrumentação de programas de ação cujo objetivo final seria a obtenção de padrões adequados de alimentação e nutrição da coletividade.

Todovia, aceita-se, por razões históricas, institucionais ou operacionais, a existência de componentes com "identidade própria", ou seja, canais bem definidos de coleta, análise e uso das informações, que devem ser complementares entre si, mesmo mantendo a particularidade de seus objetivos e procedimentos. Neste aspecto, deve ser ressaltada a recomendação específica de que a análise dos dados, além da instância setorial, deva ser objetivo de uma apreciação multissetorial, principalmente a nível central, de forma a possibilitar uma visão política e técnica conjunta dos problemas e das soluções a serem prescritas.

Em termos ideais, os vários componentes do sistema deveriam derivar de um modelo teórico-conceitual configurado segundo as características de cada situação nacional, regional ou local (problemas, fatores, existência de informações e sua períodicidade). O modelo de relações causais do estado de nutrição, proposto por Pradilla e desenvolvido por Béghin \& Noujardin (1988), representa uma boa fundamentação neste sentido.

Os componentes do Sistema de Vigilância Nutricional (Sisvan) são delineados em função de seus objetivos, como a seguir:

1. Vigilância da segurança alimentar, proposta como um instrumento informativo sobre a disponibilidade, acesso físico e acesso econômico aos alimentos básicos, segundo os padrões culturais de cada país, região e ou comunidade. Está ligada, basicamente, à definição de macropolíticas econômicas e sociais.

2. Sistema de alerta rápido, destinado a registrar ou prever crises alimentares agudas, resultantes de acontecimentos climáticos (secas, inundações, geadas, pragas estacionais) ou sociais (conflitos armados ou diplomáticos, por exemplo). O sistema está atento, portanto, a acontecimentos conjunturais, ligando-se, em primeira instância, às instituições e serviços nacionais ou internacionais de atendimento emergencial.

3. Vigilância do crescimento. Incorporada aos serviços de saúde, trabalha, em essência, com variáveis antropométricas (peso ao 
nascer, peso/idade, peso/altura, altura/idade), possibilitando acompanhar, a nível de indivíduos e comunidades, o crescimento físico das crianças e dos fatores patológicos e amambientais que interferem no processo. Constitui o mais difundido dos componentes do sistema.

\section{Vigilância das medidas de ajuste econômi-}

co, objetivando alertar os governos para a adoção de medidas de ajustes estruturais da economia, face às possíveis implicações adversas em relação à área de alimentação e nutrição. A iniciativa resultou da constatação das graves conseqüências que sofreram muitos paises do Terceiro Mundo, em razão das medidas de ajuste adotadas na vigência da recessão econômica de 1980-83.

Recentemente, propôs-se uma nova função para a VAN: a chamada "advocacia", definida como o papel político de conscientização dos governos e da sociedade sobre os problemas sociais da alimentação e da nutrição. O destaque e, sobretudo, a terminologia empregada não definem, de fato, uma nova atribuição do sistema. Apenas ressaltam, com uma referência explícita, sua função política, que deve ser exercida mediante um conjunto de estratégias: debates públicos, acesso aos movimentos sociais organizados, difusão de informes junto aos meios de comunicação de massa e outros recursos.

\section{O SISVAN NO BRASIL}

As primeiras experiências na vigilância alimentar e nutricional no Brasil foram efetuadas nos estados da Paraíba (Vale do Piancó) e Pernambuco (zona metropolitana do Recife), entre 1983 e 1984, por iniciativa do Instituto Nacional Alimentação e Nutrição (Inan). Os dois projetos, que reuniam unidades de saúde de 4 localidades estaduais (Paraíba) e 8 postos de atendimento médico do Instituto Nacional de Assistência Médica da Previdência Social (Inamps) (Recife), não sobreviveram à fase experimental, sendo desativados no momento em que o Instituto Nacional de Alimentação interrompeu o fluxo de financiamento destinado à execução desta fase.
Segue-se uma nova etapa, com o apoio do Fundo das Nações Unidas para a Infância (Unicef), possibilitando o surgimento de três projetos, nos estados de Pernambuco (Fundação Serviços de Saúde Pública), São Paulo (Secretaria Estadual de Saúde) e Ceará (Instituto de Planejamento do Estado do Ceará). Concomitantemente, a Escola Nacional de Saúde Pública (Ensp), com a ajuda do Conselho Nacional de Desenvolvimento Científico e Tecnológico (CNPq) e do Programa de Apoio à Reforma Sanitária (Pares), inicia a implantação de um projeto experimental na área de Manguinhos. Estes eventos são de considerável importância estratégica, na medida em que assinalam um compromisso interno das próprias instituições governamentais com a VAN, em oposição ao interesse "externo" do momento anterior, quando o projeto confundia-se como uma proposta de propriedade única do Instituto Nacional de Alimentação e Nutrição.

Atualmente, o Sisvan está operando em fase de implantação nos estados da Paraíba, Alagoas, Paraná, Goiás, Rio Grande do Sul, Sergipe, Maranhão e Bahia, além das quatro unidades federativas previamente citadas. Mediante a ação da Pastoral da Saúde, a Conferência Nacional dos Bispos do Brasil está difundindo a rede de informações sobre alimentação e nutrição para todas as unidades federativas, tendo como suporte de trabalho o nível comunitário. A Fundação Nacional de Saúde (ex-FSESP) também se propõe a difundir o Sistema nos diversos estados.

A partir de 1990, o Sisvan passou a ser uma atividade formal do Ministério da Saúde, mediante portaria que institui e define as atribuições do Sistema. A estrutura e funções do Sisvan nacional acham-se especificadas na portaria de sua criação.

\section{Análise da Experiência}

Tratando-se de uma atividade ainda em fase de desenvolvimento conceitual e operativo, com uma experiência muito breve no Brasil, a análise da Sisvan necessariamente representa um exercício preliminar, mais indicativo que conclusivo. É nesta direção (e sob estes limites) que se orientam as considerações expostas a seguir. 
1. Ainda é pouco consistente a sustentação política do SISVAN. As autoridades e a opinião pública não se aperceberam plenamente de sua importância. A atividade é mais consentida do que estimulada. Os indicadores mais tradicionais da qualidade de vida coletiva (mortalidade infantil, taxa de analfabetismo e de desemprego, índices de custo de vida, produto interno bruto, entre outros) têm um espaço culturalmente bem definido no tratamento dos problemas econômicos e sociais, de modo que a VAN tornase um trabalho de diletantismo técnico, ainda sem a necessária acústica política junto às autoridades e ao público. Esta é uma tarefa de "advocacia" que compete ao próprio Sisvan desenvolver e consolidar.

2. O Sisvan praticamente se resume às informações antropométricas, funcionando como um elemento de registro contínuo do crescimento de crianças nos 5 primeiros anos de vida. É um instrumento limitado, mesmo para o setor saúde. Vale como ponto de partida, mas é inquestionável a necessidade de que sejam incluídos outros indicadores sobre outros problemas (anemias, hipovitaminose A, bócio), a respeito dos quais existem projetos e atividades de prevenção ou de tratamento. Como visão prospectiva, a VAN deve abarcar o setor de alimentos (qualidade, quantidade, consumo), bem como a análise integrada, dentro de um modelo causal racionalmente concebido e validado, dos fatores mais relevantes que determinam a situação alimentar e nutricional da população (Field, 1983; Batista-Filho, 1989).

3. A cobertura geográfica e populacional do Sisvan é ainda muito reduzida, com exceção de alguns projetos (áreas faveladas do Rio de Janeiro e do Recife) nos quais mais de 90\% dos grupos alvos estão sendo continuamente avaliados. Por outro lado, com pequenas exceções, a qualidade dos dados é duvidosa. Este é um aspecto que deve ser obstinadamente trabalhado pelos profissionais que desenvolvem projetos de vigilância (BatistaFilho et al., 1985).

4. O esforço do reduzido número de técnicos na geração e processamento de dados e as próprias dúvidas sobre a consistência das informações têm deixado em segundo plano o componente de análise e interpretação dos resultados. O trabalho estatístico de construção de gráficos e tabelas domina, claramente, o esforço intelectual no sentido de compreender seu significado técnico e suas implicações políticas e programáticas. É admissível esta conduta no advento de uma experiência, mas é pertinente advertir para o fato, evitando-se a repetição do "estaticismo" pouco consequente que marca, de forma desfavorável, a burocracia técnica do setor saúde.

5. Reconhece-se, até mesmo como um desdobramento lógico dos aspectos anteriores, que o Sisvan tem pouca utilização. Se se avalia o que se espera do Sistema (fundamentação técnica para o planejamento de medidas ancoradas numa segura concepção política), é conclusiva a observação de que os dados produzidos praticamente não acionam intervenções dos governos nem reações do público. Até mesmo o "circuito menor" da VAN, ou seja, o uso imediato e individual da informação pelo profissional que presta assistência, tem sido descurado na grande maioria dos casos. Não existe o necessário feedback entre as informações, a ação e seus resultados finais.

Este aspecto restritivo não é um mal próprio do caso brasileiro, ocorrendo, com raras exceções (Cuba, Chile e Costa Rica, por exemplo), na quase totalidade dos países latino-americanos. Ainda que desculpável, por uma soma de grandes razões, a subutilização dos dados deve constituir um problema permanente a ser enfrentado, na medida em que, sem a ação oportuna e competente, o próprio conceito de vigilância passa a ser comprometido e, até mesmo, desautorizado.

\section{CONSIDERAÇÕES FINAIS}

1. É possível que os comentários anteriores possam ser tomados como negativos ou pessimistas, na medida em que assinalam desafios de grande porte para uma massa reduzida de técnicos efetivamente comprometidos com o Sisvan. 
Torna-se evidente que, na área social, a seqüência informação/decisão/execução/avaliação de resultados constitui um ideal técnico e político que deve ser buscado sempre, mesmo que não seja alcançado logo. Existe um grande número de variáveis internas e externas que interferem com a cadeia, constituindo um papel próprio do Sistema procurar o máximo de controle das primeiras, em consonância com um mínimo de articulação com as segundas. Esta conduta reduz os gaps, os deficits de fluxo, mesmo sem se alcançar o objetivo idealista de um sistema perfeito (FAO, 1989).

Ademais, dentro do alcance da VAN (conforme se depreende de seus objetivos e modalidades), acha-se potencialmente subentendido um conteúdo político e social que deve constituir um compromisso de ação permanente dos governos e das sociedades, no sentido de se alcançar níveis qualitativos de vida à altura dos recursos naturais existentes, dos conhecimentos científicos disponíveis e dos instrumentos técnicos que possibilitam sua materialização. $\mathrm{O}$ Sisvan é uma estratégia em constante aperfeiçoamento e mutação, na medida em que se refere e se luta para que os objetivos finais da alimentação e da nutrição sejam componentes integrantes das grandes prioridades políticas e dos programas governamentais legitimados pelo consenso da sociedade (Carlson \& Wardlaw, 1990).

Dentro deste enfoque, o imediatismo dos problemas e das soluções não deve restringir o andamento do objetivo maior do Sistema: o acesso universal aos bens materiais e aos serviços básicos, entre os quais o componente alimentar/nutricional assume uma conotação imperiosa de ética social do próprio desenvolvimento.

\section{2. É necessário um esforço imediato de marke-} ting no sentido de assegurar uma acústica política básica à proposta do Sisvan, de forma a superar a conotação de um projeto puramente técnico. É urgente, ainda, a tarefa de se obter um mínimo de padronização dos diversos projetos. Uma vez que as experiências mais antigas tendem a se constituir como pólos de referência para a replicação do sistema, diferenças marcantes (sobretudo no que se refere aos indicadores utilizados) poderiam propor- cionar uma grande diversidade de modelos informativos no País, dificultando o tratamento de seus resultados.

3. Dentro do setor saúde, parece consensual o reconhecimento de que a VAN deve resgatar, ao máximo, o compromisso das ações básicas e integradas de saúde, valorizando os aspectos referentes à assistência pré-natal, planejamento familiar, assistência ao parto e ao recém-nascido, controle do crescimento e desenvolvimento, prevenção e tratamento das diarréias e de doenças respiratórias agudas, vacinações de rotina, promoção do aleitamento materno e orientações educativas. Os modelos de registro de atividades utilizados pela Fundação Nacional de Saúde e pela Escola Nacional de Saúde Pública representam um bom instrumento para a coleta das informações pertinentes a este tópico.

4. Um outro ponto de manifesto interesse político e técnico seria a inclusão dos dados mensais sobre o preço da cesta básica de alimentos e seu cotejo com a pirâmide de distribuição de renda no Brasil. Este indicador poderia avaliar o percentual de famílias que teoricamente não têm acesso econômico a um elenco de produtos que compõem as necessidades alimentares básicas da população. É oportuno referir que várias instituições (DIEESE, Instituto Brasileiro de Geografia e Estatística (IBGE), Fundação Getúlio Vargas (FGV), entre outras) há vários anos produzem estas informações, tornando-se indesculpável o não-aproveitamento de seus resultados pelo Sisvan. A "versão nutricional" da cesta básica, avaliada por enquanto, como um simples indicador econômico, constitui uma responsabilidade formal a ser assumida pelos que trabalham na área de alimentação e nutrição no País. Seria um bom ponto de partida para a concepção de um sistema de segurança alimentar (Lechtig, 1990; Mason et al., 1984).

5. Para o monitoramento das carências nutricionais específicas (anemia, bócio, hipovitaminose A e, possivelmente, a cárie dental por deficiência de flúor), existem possibilidades concretas que podem ser proveitosamente trabalhadas. Do 
grupo de carências específicas, a situação da vitamina A constitui o problema de abordagem mais difícil em termos de vigilância.

6. A inclusão da Pastoral da Saúde e da Criança, que mobiliza dezenas de milhares de agentes e voluntários por todo o País, franqueia a possibilidade de se desenvolver um trabalho junto às comunidades, difundindo o conceito e as práticas da VAN junto às populações de difícil acesso físico e social para as instituições de serviço público. É um excelente contraponto para um trabalho participativo e crítico, em contraste com o caráter paternalista que ainda fundamenta a ação dos serviços assistenciais mantidos pelos governos.

Parecem ser estas, de imediato, as indicações mais relevantes para o desenvolvimento da VAN no País. Está implícita a necessidade de formação de um forte contigente de recursos humanos, de forma a constituir uma massa crítica para promover e apoiar um elenco de iniciativas e desdobramentos que delineiam-se para a implantação de um sistema conseqüente de vigilância alimentar e nutricional.

\section{RESUMO}

BATISTA-FILHO, M. \& RISSIN, A.

Vigilância Alimentar e Nutricional: Antecedentes, Objetivos e Modalidades. A VAN no Brasil. Cad. Saúde Públ., Rio de Janeiro, 9 (suplemento 1): 99-105, 1993.

Os autores descrevem os conceitos, objetivos, componentes e ações estratégicas mais recomendados para o desenvolvimento dos sistemas de vigilância alimentar e nutricional, destacando a importância de um processo contínuo de geração, processamento e análise de dados para o acompanhamento da situação alimentar e nutricional e suas tendências temporais, espaciais e sociais. O Sistema de Vigilância Nutricional (Sisvan) seria um instrumento indispensável para o planejamento, implantação e análise de políticas e programas destinados ao alcance das metas internacionais (Unicef/FAO/OMS) de saúde e nutrição para o ano 2000.
Analisam-se a evolução e a situação atual dos sistemas de vigilância nutricional no Brasil, relacionando-se os principais problemas (cobertura, indicadores, capacidade de análise e usos) que devem ser enfrentados para a consolidação do processo.

Palavras-Chave: Vigilância Nutricional; Alimentos

\section{REFERÊNCIAS BIBLIOGRÁFICAS}

ARANDA-PASTOR, J. \& KEVANY, J. P., 1980. Establecimiento de sistemas de vigilancia alimentaria y nutricional. Contribución del sector salud. Boletín de la Oficina Sanitaria Panamericana, 89: 473-479.

BATISTA-FILHO, M.; LUCENA, M. A. F. \& SOUZA, M. C., 1985. A vigilância alimentar e nutricional no Brasil. II Mesa Redonda Internacional sobre Sistema de Vigilância Alimentar e Nutricional na América Latina. Bogotá: FAO/ OPS. (Mimeo.)

BATISTA-FILHO, M. \& SHIRAIWA, T., 1989. Indicadores de saúde para um sistema de vigilância nutricional. Cadernos de Saúde Pública, 5: 105-116.

BÉGHIN, I. \& NOUJARDIN, T., 1988. A Guide to Nutritional A ssessment. Genebra: World Health Organization.

CARLSON, B. \& WARDLAW, T. M., 1990. A Global, Regional and Country Assessment of Child Malnutrition. New York: Unicef. (Unicef Staff Working Papers, 7)

CHAVEZ, A., 1989. La vigilancia nutricional en América Latina y el Caribe. Progresos durante los últimos 30 años. In: OPAS - Vigilancia Alimentaria y Nutricional en las Américas (OPAS), pp. 15-18, Washington, DC: OPAS. (Publicación Científica, 516).

FAO (Food and Agriculture Organization) (Organización de las Naciones Unidas para la Agricultura y la Alimentación), 1974. Conferencia Mundial de los A limentos. Roma: FAO. , 1989. Manual Práctico para la Instalación de un Sistema de Vigilancia Alimentaria y Nutricional. Santiago de Chile: FAO.

FIELD, J. O., 1983. The importance of context: nutrition planning and development reconsidered. In: Nutrition in the Community (D. S. McLaren, ed.), pp. 15-22, Chichester: D. S. McLaren.

ICNND (Interdepartmental Committee on Nutrition for National Defense), 1963. M anual for Nutrition Surveys. Washington, DC: ICNND. 
JELLIFFE, D. B., 1968. Evaluación del Estado de Nutrición de la Comunidad. Genebra: OMS. (Serie de Monografias, 53)

LECHTIG, A., 1990. Inquéritos nutricionais. Fundamentação. Implementação para VAN: Inquéritos de orçamento familiar, alimentos básicos. In: Seminário Internacional sobre Sistemas de Vigilância A limentar e Nutricional em Países A fricanos de Língua Oficial Portuguesa (FAO/OMS/ UNICEF, orgs.), p. 4 , DT./06, Maputo, Moçambique. (Mimeo.)

MASON, J. B.; HABICHT, J. P.; TABATABAI, H. \& VALVERDE, V., 1984. Vigilancia Nutricional. Genebra: Organización Mundial de la Salud.

MASON, J. B., 1984. Proposed guidelines for designing evaluation for nutrition and health programmes. Food and Nutrition Bulletin, 6: $11-23$.
OMS (Organización Mundial de la Salud), 1978. Atención Primaria de Salud. Genebra: OMS. (Serie Salud para Todos, 1)

OPS (Organización Panamericana de la Salud), 1989. Vigilancia Alimentaria y Nutricional en las A méricas. Washington, DC: OPS. (Publicación Científica, 516)

UNICEF (Fundo das Nações Unidas para a Infância), 1990. Estratégia para Melhorar a Nutrição de Crianças e Mulheres nos Países em Desenvolvimento. New York: UNICEF. 